\title{
Editorial
}

\section{Cher lecteur,}

Le premier volume de Ressources Génétiques Animales fut publié en 1983, sous le nom à cette époque de Bulletin d'Information sur les Ressources Génétiques Animales. Maintenant, 33 ans plus tard, le journal arrive à son terme avec son $59^{\text {ème }}$ volume, et ce principalement en raison du manque de ressources. Dans l'éditorial du volume commémoratif 50 quelques informations sur l'histoire du journal et quelques données statistiques vous étaient déjà fournies. Je voudrais maintenant partager avec vous les chiffres finals.

Le $50^{\text {ème }}$ volume a été mis en ligne à la mi-2012. Jusqu'à cette date plus de 400 articles avaient été publiés. Dès lors, 123 autres articles ont été acceptés pour publication. Alors qu'au cours des 50 premiers volumes, le pourcentage d'articles publiés en anglais était inférieur à 80 pour cent, ce pourcentage a augmenté à près de 90 pour cent dans les neuf derniers volumes. En ce qui concerne les espèces d'animaux d'élevage, l'analyse des articles révèle que la plupart des publications continuent de se concentrer sur les ruminants. Plus précisément, dans ces neuf derniers volumes, les deux tiers des publications ont traité des petits ou des grands ruminants, à parts égales entre eux, tandis que les articles consacrés aux volailles n'ont représenté qu'un cinquième et ceux dédiés aux porcs et aux équidés une proportion encore plus faible. Bien que ce résultat confirme le rôle important des ruminants pour la subsistance des êtres humains, il peut également être interprété comme un appel à davantage de recherches sur d'autres espèces animales jouant un rôle essentiel dans certaines régions du monde, qui pourraient devenir plus importantes, étant donné les conditions environnementales changeantes.

Les articles publiés dans ce journal peuvent être mis en rapport avec plusieurs domaines prioritaires du Plan d'Action Mondial pour les Ressources Zoogénétiques $(P A M)^{1}$. Je souhaiterais rappeler que la communauté internationale a adopté le PAM à la Conférence Technique Internationale sur les Ressources Zoogénétiques pour l'Alimentation et l'Agriculture en septembre 2007. Le PAM a ensuite été approuvé par la $34^{\text {ème }}$ Conférence de la FAO. Il inclut 23 priorités stratégiques d'action regroupées en quatre domaines prioritaires: caractérisation et surveillance; utilisation durable et mise en valeur; conservation; et politiques, institutions et renforcement des capacités. Le Deuxième Rapport sur l'État des Ressources Zoogénétiques pour l'Alimentation et l'Agriculture dans le Monde $^{2}$ (publié en 2015) a fourni la base pour une révision et une possible mise à jour du PAM. Ceci confirme que les quatre domaines prioritaires sont toujours valables. Une grande majorité des articles du journal traitent du

http://www.fao.org/docrep/010/a1404f/a1404f00.htm

'http://www.fao.org/publications/sowangr/fr/ premier domaine prioritaire. Alors que 63 pour cent de tous les articles publiés dans les 50 premiers volumes s'inscrivaient dans ce domaine prioritaire, ce pourcentage est passé à 73 pour cent dans les neuf derniers volumes. Seize pour cent des articles publiés après le $50^{\text {ème }}$ volume ont traité de l'utilisation durable et la mise en valeur, contre 11 pour cent auparavant. Le pourcentage d'articles dédiés à la conservation a diminué de 17 pour cent à 8 pour cent et seul un faible pourcentage d'articles ont traité du quatrième domaine prioritaire sur les politiques, les institutions et le renforcement des capacités. Cette répartition inégale entre les domaines prioritaires peut s'expliquer par la faisabilité de certaines études soutenues par des projets de recherche limités en termes de durée et de financement, ainsi que par l'absence fréquente de programmes de sélection structurés et des données connexes.

Au total, trois volumes spéciaux du journal ont été publiés. Le dernier, intitulé “Ajouter de la valeur", a été publié en 2013. Les articles sur ce sujet décrivent plusieurs stratégies qui ont été utilisées pour ajouter de la valeur aux races locales dans divers pays et régions, afin de rendre ces races plus compétitives et de promouvoir leur utilisation durable à long terme.

Depuis 2009, la FAO a publié le journal conjointement avec Cambridge University Press. Tous les volumes et articles continueront d'être disponibles par l'intermédiaire de Cambridge Journals Online et sur le site Internet de la FAO http://www. fao.org/AG/AGAInfo/programmes/fr/genetics/journal.html.

Je tiens à remercier les lecteurs de ce journal pour leur fidélité et leur intérêt pendant tant d'années et les auteurs pour leurs précieuses contributions. Vous avez fait de ce journal une source passionnante d'informations pour tous ceux intéressés par la diversité des ressources zoogénétiques et leurs multiples produits et services. Nous espérons sincèrement que les anciens auteurs de Ressources Génétiques Animales trouveront un endroit approprié dans le vaste domaine des journaux consacrés à l'élevage pour y envoyer leurs futures publications et continuer ainsi à contribuer à la diffusion des connaissances sur les ressources zoogénétiques.

Dernier point mais non le moindre, je voudrais également remercier les relecteurs et les membres du comité de rédaction du journal, qui ont fait un excellent travail pendant de nombreuses années, en prenant au sérieux le rôle de renforcement des capacités du journal. Cela s'est reflété dans le processus de révision, qui visait, le cas échéant, à soutenir et conseiller les auteurs qui autrement auraient pu avoir du mal à présenter leur travail sous une forme appropriée pour la publication dans une revue scientifique.

Cordialement, Roswitha Baumung 